\title{
The Effects of Childhood Trauma on Drug Use
}

\author{
Geetha Amarasinghe ${ }^{1}$, Dr. Amanda Lash ${ }^{2}$, and Dr. Leonard Ranasinghe ${ }^{3 *}$ \\ ${ }^{1}$ Graduate of Whittier College Class of 2019, USA. \\ ${ }^{2}$ Assistant Professor of Psychological Sciences (2016 to 2019), Whittier College, USA \\ ${ }^{3}$ Professor of California Northstate University College of Medicine, USA
}

*Corresponding Author: Leonard Ranasinghe,Professor of California Northstate University College of Medicine, 9700 W. Taron Dr. Elk Grove, CA 95757, USA.

\section{Received Date: May 25, 2020; Accepted Date: June 11, 2020; Published Date: June 16, 2020.}

Citation: Geetha A., Dr. Amanda L., and Leonard R.(2020) Laparoscopic Diagnosis and Treatment of Stage 4 endometriosis After Traumatic Agni karma with Abdominal Scars: a Case Report.: Observational study. Obstetrics Genecology and Reproductive Sciences, 4(1): DOI: 10.31579/2690$8794 / 019$

Copyright: (C) 2020. Leonard Ranasinghe. This is an open-access article distributed under the terms of the Creative Commons Attribution License, which permits unrestricted use, distribution, and reproduction in any medium, provided the original author and source are credited.

\begin{abstract}
Exposure to childhood physical abuse, sexual abuse, emotional abuse, and neglect have been found to have a positive association with drug use. Various studies have demonstrated how individuals may turn to drugs as a form of coping. However, this is a negative form of coping, which can take a toll on one's mental state and even impact families and society. Studies have reported that a higher number of adverse childhood experiences (ACEs) have a stronger association with substance use later in life [1,3]. Recent research has also demonstrated those who experienced childhood neglect also displayed anxiety or depression, which might suggest a reason for the connection between the negligence and drug use [14]. There is also research that shows that childhood maltreatment could influence one's susceptibility to drugs and the structural remodeling of the brain [4]. Various types of drugs have been used in association with childhood trauma; however, no single drug was reported to have a greater association than the others. Substance abuse has been a rising issue over the years. With the understanding that childhood trauma could contribute to the onset of drug use, it is essential to gain a better knowledge of the specific types of substance abuse linked to childhood trauma. Future examination of this topic can help raise awareness and educate society on the effects of childhood trauma and how to prevent drug use associated with it.
\end{abstract}

Keywords: adverse childhood experiences (aces); physical abuse; sexual abuse; emotional abuse; neglect; substance use; drugs; alcohol; smoking

Abbreviations: ACEs: Adverse Childhood Experiences; PTSD: post-traumatic stress disorder; OFC: orbitofrontal cortex; NLAAS: National Latino and Asian American Study; BRFSS: Behavioral Risk Factor Surveillance System; MRI: Magnetic resonance imaging; M.I.N.I: Mini-International Neuropsychiatric Interview

\section{Introduction}

In the United States, the majority of children encounter one or more forms of trauma, and it is estimated that $90 \%$ of people endure at least one traumatic event during their life [22, 26]. Those who face adverse childhood experiences (ACE) have an increased likelihood of lifetime substance use disorders and a higher risk for alcoholism, depressed affect, post-traumatic stress disorder (PTSD), and illicit drug use [12,26].

Childhood trauma could also contribute to neurodevelopmental issues and promote impaired constraint for drug use [4]. These neurological changes can lead to a significant reduction in grey matter concentration of the orbitofrontal cortex (OFC), which is a part of the brain that functions as a mediator for cognitive and impulse control behaviors, as well as reward processing $[4,19,20]$. The changes in the brain may contribute to the tendency for ACE victims to turn to drugs to achieve pleasant sensations, due to their inhibited impulse control and the need to abandon negative emotions. Individuals who face ACEs may have issues managing their environmental stress due to neurological changes in the brain. In turn, this would make it more difficult for them to respond effectively in stressful situations, leading them to turn to drugs to relieve tension $[15,16]$.
Experiences of trauma during the early years could affect the outcome of certain mental developments, contributing to a dependency on drugs later in life [12]. Exposure to childhood adversity leads to about $64 \%$ of the population-attributed risk for illicit drug use [12]. In the United States, there is a high rate of alcohol and cannabis use, as well as the use of opioids and other substances, which in many cases link to some form of childhood trauma [29]. There are several types of childhood trauma or adverse childhood experiences (ACE) connected to future drug use, including physical, emotional and sexual abuse, Post-traumatic Stress Disorder (PTSD), exposure to violence, neglect, and emotional dysfunction associated with alcohol or drug use [11,12,34,35]. Substance abuse might develop for individuals to cope with the pain from their childhood trauma, and exposure to childhood adversity leads to the majority of illicit drug use cases [12].

In the U.S., over 679,000 children experienced abuse and neglect in 2013 [40]. Physical abuse, sexual abuse, emotional abuse, and neglect have been found to have a positive association with drug use $[1,2$, 18]. In general, definitions of the types of abuse were similar across studies. Childhood physical abuse was identified to be associated with physical beatings or threats with a weapon, while childhood sexual abuse was defined as rape, stalking, or inappropriate touching [1,2]. Childhood 
neglect was defined as a form of abuse where a child does not receive appropriate care or isolated [30,32]. Emotional abuse, which might coincide with neglect, was identified to be a form of maltreatment causing the child to feel unloved [30,32]. It was found that $96.5 \%$ of individuals with substance use disorders had faced at least one form of trauma at some point in their life [24]. Certain types of childhood trauma may have a stronger connection to specific kinds of drug use.

One study found that physical abuse had an association with drug use [9]. The study focused on examining the differences in severity of childhood physical abuse [9]. They found that severe forms of physical abuse had a greater connection to drug use and about $74 \%$ of participants had experienced abuse and $82.7 \%$ reported a history of drug use [9]. Other studies have reported similar findings linking childhood physical abuse to a higher likelihood of specific types of drug use [3]. Another study suggests that the severity of the kind of abuse might influence the drug use susceptibility [13]. These studies, among others, highlight the prevalence of childhood trauma in our current society, as well as the possible connection to drug use.

The purpose of the current literature review is to focus on comparing the relationship between childhood trauma and drug use to evaluate the research linking ACE to the different types of drug use that arise in later years of life. Given the recency of several articles addressing the topic of childhood trauma and drug abuse there still lacks a literature review concentrating on this subject within the past five years. Therefore, this review aims to compile and analyze a variety of journal articles that have recently examined the effects of childhood trauma on drug use. This literature review will provide a thorough analysis of the topic and address inconsistencies and connections found among the various studies.

The review will begin with a brief description of the forms of trauma. This will include physical and emotional abuse and neglect and sexual abuse. This will lead to family backgrounds and contribute to childhood trauma, followed by the neurological and psychological impact of trauma. The proceeding section will focus on drug use, which results from childhood trauma. This section will cover the different types of drugs that are often abused, beginning with smoking and drinking, then other substances. It will also include a description of the effects of trauma and drug use on the brain and well-being. The review will end with a conclusion and possibilities for future research.

\section{Forms of Childhood Trauma}

Individuals under the age of 18 years exposed to physical abuse, sexual abuse, emotional abuse and/or neglect have been found to have a positive association with drug use $[1,2,18]$. Two commonly reported forms of trauma had been physical and sexual abuse, and both types have been noted to have positive associations to drug use later in life, but findings on this association have varied among studies. Additionally, experiences with emotional abuse and neglect have also been identified to have a connection to drug use, and in some cases, multiple forms of abuse might coincide. The various types of childhood trauma reported in recent studies have similar consequences that lead to drug use or misuse later in life. This section will look at the different forms of abuse, how they are related to drug use and variations among demographics.

\subsection{Physical and Sexual Abuse}

A group of researchers used the National Latino and Asian American Study (NLAAS) to examine the effects of childhood trauma [1]. They collected data through a self-report survey to evaluate the effects of childhood physical abuse and childhood sexual abuse on substance abuse behavior in adulthood among a sample of men. Nearly $36 \%$ of participants reported childhood physical abuse and about $6 \%$ reported sexual abuse. A total of $21.6 \%$ of participants reported substance abuse, either as a lifetime issue or as an occurrence within the past year. The findings suggest that childhood physical abuse had a positive association with substance abuse; however, childhood sexual abuse did not have a significant relationship with substance abuse [1]. Sexual abuse might not have a positive association with substance abuse due to the low number of self-reports of sexual abuse compared to the high number of physical abuse reports. The connection between abuse and substance abuse was suggested to be due to negative coping strategies adopted to overcome stress.

Another study focused on the effects of childhood physical and sexual abuse with an ACE survey, which included specific questions on the type of trauma experienced [3]. Participants were all Latino, and they were asked questions regarding their experiences during their first 18 years of life, and they were asked to quantify their exposure to abuse. Physical abuse was found to be one of the three most common forms of childhood trauma. On average, participants reported experiencing at least two forms of trauma during their childhood. A higher number of ACEs were found to have a stronger association with substance use [3]. Both studies reported an association between childhood physical abuse and drug use, which might be due to the similar populations sampled, as both studies focused on Latino-American men within the United States $[1,3]$. It was noted that physical abuse might be more prevalent within this ethnic group due to the normalization of physical abuse in their culture [1]. Adults with a history of traumatic childhood events might engage in drug use as a coping strategy to mask the negativity they encounter from trauma [3].

There may be a few explanations for these associations. ACEs have an impact on emotional and mental state and therefore may cause behavioral changes as well, leading the affected children to associate with the wrong peer groups or engage in risky behaviors, which in some cases involve drugs. In situations where children are exposed to caretakers who use drugs, they may be more likely to adopt the practices from them, and this might indicate an inherited susceptibility to drug use.

A different study focused on a sample of college students and utilized the American College Health Association's National College Health Assessment II to collect data on ACE and drug use [17]. Their sample was more diverse than the previous two studies, consisting of 6 different ethnic groups, with mostly Hispanic and Non-Hispanic White participants [1,3]. All participants provided information regarding their abuse history by answering a series of questions; each question was coded, with a 1 for "yes" and 0 for "no." Responses that indicated exposure to abuse resulted in a higher ACE score, which was calculated by summing responses. They found that 50 to $75 \%$ reported substance use and this had a connection to childhood adversity [17]. In this case, sexual abuse was reported more frequently than physical abuse, which contrasts the results of previous studies $[1,3]$. The current study used a sample of $73 \%$ female and they had higher reports of sexual abuse and a significant association between abuse and drug use [17]. This differs from the studies that focused on all-male subjects $[1,3]$.

This difference may suggest that sexual abuse is more common among females or that females are more likely to report sexual abuse compared to men. However, one group of researchers used a series of assessments to collect data and they found that there was weak evidence of any gender differences when it came to sexual abuse histories and drug use [18]. This study is different from the previously mentioned studies, since this study used a larger sample, which included about 20 times the number of participants [18]. This more ample sample might account for the variation in data and therefore the similar reporting between males and females. Additionally, this study asked multiple questions related to physical and sexual abuse, and instead of having participants strictly answer "yes" or "no," as in the previous study, they were rated on a scale depending on their answers of the ACE assessment questions [18]. For instance, participants answered questions about the number of times they 
experienced abuse, and researchers rated them on the severity of their experience, rather than the occurrence of ACE alone. This method might have provided more accurate results when measuring the frequency of physical and sexual abuse concerning drug use.

Data from NESARC, a representative epidemiological study of the adults in the U.S was used in a study to examine the effects of abuse [29]. Participants completed a series of questions from the Conflict Tactics Scale, and they created a composite variable to assess the amount of childhood abuse participants experienced as children. Drug use was also recorded through a questionnaire. They found that there was a relationship between exposure to abuse and future drug use, but gender was also identified to have little significance [29]. This finding could mean that individuals who face childhood adversity turn to drugs as a coping mechanism. However, the type of adversity and how it is experienced also needs to be considered.

Data collected from a sample of Canadian respondents was used to examine the relationship between childhood sexual abuse and substance abuse [38]. Participants had a wide age range of 18 to 76 years, and they completed both surveys and interviews to provide ACE history and drug use status. Reports showed that $14 \%$ of women had been exposed to sexual abuse, while only $5 \%$ of men experienced sexual abuse. This finding aligns with previous studies on U.S. participants [17]. Results also showed that gender differences were negligible when it came to associations between childhood sexual abuse and substance abuse. This result might suggest that even though women and men experience physical and sexual abuse at different rates, they can both turn to drug use regardless of the type of abuse suffered. This finding is complementing to two previous articles, which might imply that there may not be a difference between males and females when it comes to reacting to childhood abuse and adapting substance use behaviors [18,29]. Overall, it was found that there was little significance in the relationship between childhood trauma and substance abuse, which might have been due to the age gap in their sample [39]. The older respondents may not have been able to recall their past experiences of trauma accurately, additionally coming from a different generation drug use might not have been as common as it is now, for younger participants. This could mean that the data was not an accurate representation of childhood trauma and drug use in the current society, rather a blend of data from different generations. The generational and cultural differences between the older and younger participants might have contributed to the scattered data and low significance.

Additionally, a different study examined a population of men and women, and participants completed a substance use interview, where researchers gaged their involvement with drugs and after they completed a childhood trauma questionnaire [42]. They also found that both genders were likely to engage in drug use, following childhood physical and sexual abuse, which is similar to the findings of two previously mentioned articles $[18,29]$. The results displayed that women had encountered more experiences with childhood sexual abuse and men had experienced more childhood physical abuse; however, in both cases, men and women with ACEs had a positive association with drug use. This finding further supports the idea that regardless of gender, people have similar reactions to childhood trauma, and turning to drugs seems to be the most popular way of dealing with these types of traumatic occurrences.

Physical and sexual abuse has been shown to lead to drug use during adulthood in various samples of people, and two other forms of childhood trauma are often reported to connect to future drug use, emotional abuse, and neglect.

\subsection{Emotional Abuse and Neglect}

Child neglect is a common type of stressor that a child might face, and it can potentially impact development and result in drug use [14]. In one study, researchers concentrated on childhood neglect, as it is often experienced alone, without other forms of abuse [14]. They focused on neglect in terms of failure-to-provide and a lack-of-supervision for the child. The Longitudinal Studies on Child Abuse and Neglect was used to account for the lack of research that longitudinally traces associations between childhood neglect and substance use [14]. As a part of the data collection, the child's caregiver was interviewed to gain a background of the child's relationship with the person. This process could have provided researchers with biased results, as caregivers might not express child neglect as an issue during their interview. However, researchers also used Child Protective Service records which provided a history of each individual's childhood neglect. Results showed that childhood neglect, especially during the first four years of life, had a positive relationship with the development of drug use [14]. The article also reported that those who experienced childhood neglect also displayed internalizing symptoms, such as anxiety or depression, which might suggest a reason for the connection between the negligence and drug use [14]. That is, one might feel more inclined to use drugs to mask the symptoms of anxiety or depression related to their experience of neglect.

One study gathered a sample of men and women to gather information regarding their childhood experiences and drug use behaviors [38]. All participants agreed to partake in the NAOMI heroin-assisted opiate dependence treatment study for heroin users ages 25 years and older. The results demonstrated that childhood neglect and emotional abuse had a stronger relationship with early drug initiation [38]. Over 70\% of subjects in this sample reported either emotional abuse or neglect, and individuals with a family history of drug use were more likely to turn to drugs themselves. This finding might suggest that people are influenced by the behaviors they witness as a child, as those who grow up with guardians who use drugs are likely also to use substances in their future. The study also identified patterns in the types of abuse; maternal alcohol abuse was associated with all forms of trauma except physical abuse, and paternal alcohol abuse was associated with physical abuse and neglect [38].

Another group of researchers also focused on child neglect and drug use and utilized the National Longitudinal Study of Adolescent to Adult Health to examine how childhood neglect would impact self-esteem [30]. The longitudinal design was beneficial in monitoring changes in an individual's self-esteem throughout the years. They found that those who experienced neglect had varying levels of self-esteem, and those who reported a decrease in self-esteem over the years were more likely to abuse substances [30]. Low self-esteem might indicate that the individuals house negative feelings towards themselves, likely due to their exposure to child neglect. The negative feelings might prompt them to use drugs to distract from their childhood trauma. The low levels of self-esteem may arise from the lack of care and attention provided by the individual's caretaker during their childhood, which might have had an effect on their self-image and thus led to an increased risk for substance use and abuse.

Another study found a similar connection between changes in internalized emotions and substance use in individuals who experienced emotional abuse during their youth [27]. Their study relied on selfreported data and questioned participants on their childhood trauma history, as well as emotional dysregulation, which is the inability to effectively control one's emotional response in a stressful situation [27]. Those who experienced widespread abuse had higher levels of emotional dysregulation, and this was found to hold for all types of abuse, including emotional abuse [27]. Likewise, lifetime drug use was also tied to emotional dysregulation abuse [27]. This finding is similar to results of a previous study, as they showed that both neglect and emotional abuse 
impact one's development and can lead to issues with regulating emotions, thus leading to the use of drugs in an attempt to soothe negative feelings $[14,27]$.

If individuals can avoid emotional dysregulation, they may be less likely to develop substance use disorders. Researchers conducted a study to examine the effects of a protective adult relationship on a child with traumatic experiences [6]. The study used a series of surveys, including the Longitudinal Studies of Child Abuse and Neglect, where youths answered questions regarding their relationships with the adults in their life [6]. They found that those who did not have at least one healthy adult relationship were more likely to develop substance abuse issues, while those who did have a protective adult relationship were able to moderate the link between trauma and drug use [6]. This finding demonstrates the importance of adult relationships during childhood, and it shows that there may be an alternative to seeking drugs to ease the pains of child abuse, as adequate emotional support can potentially prevent substance use. This is likely because the supportive relationship allowed for youths to develop a normal emotional regulation pattern, allowing them to cope with their issues with the assistance of the protective adult, rather than drugs.

As seen from these studies, childhood trauma can lead to various issues later in life, and drug use may result as a means to temporarily resolve the pain or other unpleasant emotions. For this reason, it is crucial to understand that the connection between childhood trauma and drug use involves other contributing factors. However, almost all of these studies relied on self-reported data, which might not always be the most accurate way of addressing this research topic, since some individuals might not be willing to express all of their experiences with abuse or as an adult, they might not be able to recall their abusive experience accurately. At the same time, individuals may not always report experiencing anxiety or related depression disorders, and this might contribute to the variation in data across different studies. The following section will focus on the specific types of drug use associated with childhood trauma and how those drugs might affect well-being.

\section{Forms of Drug Use}

Based on the self-medication hypothesis, individuals who endure stressful or traumatic events may resort to drugs to disassociate from the negative feelings associated with the experience [21]. While many adults face substance abuse issues, children who suffer adverse experiences are also susceptible to developing substance abuse issues [2]. If issues with substance abuse are not corrected, they can continue through adulthood, and drug use may become a physiological need, leaving individuals more susceptible to addiction [3]. Problems associated with drug abuse, not only pose a threat to individuals involved with the substances, but they can also put a risk to families and communities [8].

In the year 2000, about 85,000 deaths ascribed to alcohol use, and about 17,000 deaths linked to the use of illicit substances [28]. Reported statistics show that over 27 million people have used illicit drugs, and approximately 66 million reported misuses of alcohol, in the United States alone [7]. Common types of substance abuse include drinking and smoking, as well as illicit drug use, all of which can have detrimental effects on mental health and overall well-being. Various studies on childhood trauma indicate the reporting of different types of drug use. Recent studies have demonstrated the possible relationship between specific types of childhood trauma and drug use, as well as affiliated consequences.

\subsection{Alcohol and Smoking}

One study used the 2011 Behavioral Risk Factor Surveillance System (BRFSS), which consisted of an 11-item ACE scale that measured forms of childhood abuse, to examined the effects of ACE on the use of cigarettes and smokeless tobacco products [2]. Additionally, participants were surveyed to identify the smoking habit, and it was found that those with ACEs had a greater likelihood of tobacco use [2]. However, there was no single form of abuse identified to have a stronger relationship with tobacco use [2]. This finding could denote that all measured types of trauma, including physical, sexual, and emotional, have equal effects inducing tobacco use habits or that some forms of ACE are more likely to trigger tobacco use. This could also imply that forms of abuse are interrelated. The data provided showed similar values for the number of individuals who faced one of the three kinds of abuse, and it could be that the same participants were reporting more than one form of abuse, but this is unknown since there was no measure to identify if participants had multiple adverse experiences.

Another considerable factor is the low report of recent smoking behavior. About $44 \%$ of participants reported ever smoking, but only about $18 \%$ reported that they were current smokers and less than $4 \%$ were current smokeless tobacco users [2]. This finding could suggest that more subjects with a history of ACE could have previously turned to smoke as a coping mechanism, but eventually chose to quit. There is also a possibility that participants partake in other forms of drug use, not measured in this study. Lastly, this finding may also indicate that the substance use behaviors, specifically smoking, are not always life-long, which might account for the decrease in smoking behavior

A group of researchers surveyed participants to examine types of childhood adversity, as well as the prevalence of smoking [25]. Similar to another study, they found that a greater number of individuals with childhood trauma reported being a former smoker than a current smoker $[2,25]$. These findings may confirm the idea that if ACE leads to smoking as a coping mechanism, smoking habits are not life-long. However, it was also found that in women, there was a higher association between childhood adversity and smoking, compared to men [25]. This could be due to the differences in the types of adversities experienced by men and women, meaning certain types of adversities are more likely to lead to smoking, or perhaps current smoking behaviors are more prevalent in women. This study also examined the effects of ACEs on anxiety [25]. Those with higher anxiety levels may turn to drugs to calm their nerves, and for that reason, researchers compared the anxiety levels of individuals who had ACEs [25]. They found that women also had higher levels of anxiety, which might explain why there was a higher association between adversities and smoking for women [25].

Another study examined the effects of childhood trauma and posttraumatic stress disorder (PTSD) on alcohol abuse [8]. Subjects in the study completed a battery of self-report measures, through a verbal interview. The measures included a childhood trauma questionnaire, a PTSD scale, and the Alcohol Use Disorder Identification Test, which screened for current alcohol abuse [8]. They found that trauma and PTSD were associated with problematic alcohol use; however, this effect of PTSD was more significant for men, than women [8]. This study contained a greater sample of women who reported PTSD, compared to men, and the fact that men had a greater likeliness to have alcohol abuse problems, might signify than men with a history of childhood trauma may have a higher susceptibility for problematic alcohol use. Although, this finding could also suggest that men are more openly willing to communicate their problematic alcohol use, compared to women.

A different group of researchers investigated characteristics of childhood maltreatment on adolescent marijuana use [13]. Data derived from the Longitudinal Studies of Child Abuse and Neglect and individuals reported experiences of physical abuse, sexual abuse, and neglect. Levels of marijuana use were also indicated and selected based on the frequency subjects used the drug [13]. The study showed that childhood maltreatment was positively associated with marijuana use [13]. It was also found that sexual and physical abuse had a stronger 
relationship to marijuana use, unlike a previously mentioned study, which found no significant relationship between the specific type of ACE and drug use $[1,13]$. This finding shows that childhood maltreatment is not always associated with substance use in the same way and there are other factors to consider, such as the type and timing of the experience. This difference in result may be due to skewed data, since there were a higher number of sexual and physical abuse reports compared to other forms of abuse, possibly indicating that sexual and physical abuse were more common among this sample. However, one study examined the effects of childhood maltreatment on the age of alcohol and marijuana use and they surveyed participants and also found that those who experienced sexual abuse, had higher reports of marijuana use [31].

Also, males had a higher chance of engaging in heavy marijuana use, than females [13]. This finding, suggested that men were more likely to engage in drug use, following an ACE is similar to another study, which indicated that men with childhood trauma were more likely to abuse alcohol [8]. However, it is important to note that one study in particular used an adolescent sample [13]. While the benefit to this is a more recent recollection of ACEs, the drawback may be peer influence. During adolescent years individuals can be strongly influenced by peers, as about $48 \%$ reported having peers who frequently used the drug [13]. Also, this peer influence may potentially differ in terms of gender, which might account for the gender differences. Males might have faced greater difficulty coping and receiving emotional support from peers, which might have influenced them to seek alternative methods to relieve their negative feelings. While, females might have received more emotional support, making them less likely to turn to drugs. Also, the males in this sample might have associated with or sought out peers who had access to drugs, and therefore they were more likely to use drugs, compared to females. This might suggest that childhood maltreatment does not necessarily directly connect to marijuana use. Instead, marijuana might be a readily available drug for this population, and reports of ACE have no association with it.

An additional study, focused on the associations of ACEs on alcohol abuse and used an online survey to collect their data [23]. The survey included an alcohol abuse test and an ACE questionnaire, as well as the questions on the co-occurrence of ACEs and alcohol abuse. Overall, there was a positive relationship between ACE and alcohol abuse [23]. Higher scores on the ACE questionnaire also corresponded to living with a substance abuser. This could mean that subjects might have been genetically predisposed to have substance abuse issues or they might have been influenced by the individual(s) who lived with them. Overall, most studies have demonstrated a positive relationship between ACE and drug use, but the effects of specific types of abuse on drug use remain unclear. Consequences associated with particular types of drug proceeding ACEs will be discussed in the following subsection.

\subsection{Other Substances}

One group of researchers, aimed to examine the role of substance use coping on sexual abuse [5]. Participants completed an online survey, which measured childhood trauma and substance use coping practices [5]. Results showed that a third of participants reported experiencing childhood sexual abuse and there was also an association between substance use coping and sexual abuse [5]. This implies that subjects who experienced sexual abuse turned to the use of methamphetamine to soothe negative emotions tied to the experience. While there is a clear connection between sexual abuse and methamphetamine in this study, the survey did not explore other possible types of abuse and specifically sought out participants with a history of methamphetamine use. Therefore, one cannot conclude that sexual abuse directly leads to methamphetamine use; however, it is still a possibility that the ACE did contribute to the substance use coping behavior.
Another study found that a consequence of childhood maltreatment was neurophysiological changes, associated with substance use [15]. In this experiment, participants underwent magnetic resonance imaging (MRI) to compare the effects of cocaine-craving between a group who experienced moderate abuse and a group who suffered severe abuse. The participants were provided with script-guided mental imagery of a stressful event and drug use [15]. It was found that the brain's ability to respond to stress was altered in those who had experienced childhood trauma [15]. Along with changes in stress response, ACE and cocaine use have also shown to have reduced certain areas of grey matter in the orbitofrontal cortex (OFC), necessary for higher-order cognitive functioning [4].

The study used MRI to examine the brain of cocaine users and found that those who experienced childhood trauma had diminished grey matter in the orbitofrontal portion of the brain [4]. The decrease in grey matter volume in those with substance use disorders could be due to the drugs releasing excess extracellular dopamine, a neurotransmitter that is responsible for the pleasant sensation [4]. The amounts of dopamine then surpass the dopamine-induced by the body's physiological response, and there is a decrease in dopamine receptor availability [4]. The decline in dopamine function links to the reduction of grey matter in the OFC. This connection might suggest a reason for the development of cocaine abuse, as individuals with an impaired decision-making ability may be more likely to turn to cocaine when attempting to cope. It also demonstrates the idea that childhood maltreatment could impact not only one's susceptibility to drugs but the structural remodeling of the brain.

Another study looked into the interaction between ACEs and methamphetamine use and METH-associated psychosis, which is a psychotic state that closely resembles paranoid schizophrenia [10]. Those who experience METH-associated psychosis may have delusions and auditory hallucinations. Researchers gathered participants from a rehabilitation center, and they were first surveyed to collect ACE data and to record methamphetamine use, and they were given the MiniInternational Neuropsychiatric Interview (M.I.N.I) to measure levels of psychosis [10]. This study examined different types of abuse and drug use, in contrast to the research conducted in a previous study, which only focused on sexual abuse and methamphetamine use [5]. It was found that both emotional and sexual abuse was associated with lifetime METHassociated psychosis [10]. This finding is similar to the previously mentioned study since they both found a positive relationship between sexual abuse and substance use coping $[5,10]$. This may suggest that there are changes in behavior associated with childhood trauma, as well as substance use. It also confirms the findings that there are changes that occur within certain regions of the brain, which can negatively impact functioning and feedback [4].

One study had participants complete M.I.N.I and a survey measuring drug use [16]. Subjects then completed a stress test, where they were asked to prepare a speech for an audience; the stress hormone, cortisol, was measured prior and after the speech [16]. Results indicated a positive relationship between stress reactivity, which was measured with cortisol levels, and ACE scores among individuals with cocaine dependence. This result was similar to another study which emphasizes the occurrence of neurophysiological changes due to trauma exposure [15]. This finding could imply that those who have faced ACEs and now use cocaine have greater difficulty coping with stress, therefore likely craving cocaine to cope with external pressures. Additionally, cocaine craving and addiction might be a greater issue for childhood trauma victims to overcome due to their alterations in stress reactivity.

\section{Conclusions}

In 2015, about 7.2 million children in the U.S. were reported to child protective services for neglect and child abuse [41]. Children who 
experience neglect may grow up with a greater vulnerability to risky behaviors, in comparison to children who do not have such experiences. As noted from the various studies, early trauma could increase one's risk for developing substance use disorders to mediate the emotional impact of the adversity [14]. The studies made it understandable that ACEs have a connection to the use of various types of drugs and researchers have demonstrated the negative effect trauma, and illicit substances could have on the brain, stress reactivity, and emotional regulation $[2,4,8,15,16,23,25]$.

Substance use disorders can be associated with many negative results, which impact the individual, as well as society. About 22.2 million Americans ages 12 and older suffer from substance abuse disorders, and one in 36 people abuse illicit drugs, while one in 15 abuse alcohol [18]. Clearly, there is an issue with drug use, and it is crucial to continue research on this topic, to gain a better understanding of how childhood trauma contributes to drug use. Future research may want to use more longitudinal studies to examine the effects of childhood trauma since that would allow researchers to track stages of development and examine when drug use is likely to occur. Additionally, it would be beneficial for studies to focus on ways of overcoming or avoiding drug initiation for individuals who have experienced childhood trauma.

\section{References}

1 Ai, A. L., Lee, J., Solis, A., \& Yap, C. (2016). Childhood abuse, religious involvement, and substance abuse among LatinoAmerican men in the United States. International Journal of Behavioral Medicine, 23, 764-775.

2 Alcalá, H. E., Ehrenstein, O. S., \& Tomiyama, A. J. (2016). Adverse childhood experiences and use of cigarettes and smokeless tobacco products. Journal of Community Health, 41, 969-976.

3 Allem, J., Soto, D. W., Baezconde-Garbanati, L., \& Unger, J. B. (2015). Adverse childhood experiences and substance use among Hispanic emerging adults in Southern California. Addictive Behaviors, 50, 199-204.

4 Bachi, K., Parvaz, M. A., Moeller, S. J., Gan, G., Zilverstand, A., Goldstein, R. Z., \& Alia-Klein, N. (2018). Reduced orbitofrontal gray matter concentration as a marker of premorbid childhood trauma in cocaine use disorder. Frontiers in Human Neuroscience, 12, 2-12.

5 Berg, M. K., Hobkirk, A. L., Joska, J. A., \& Meade, C. S. (2017). The role of substance use coping in the relation between childhood sexual abuse and depression among methamphetamine users in South Africa. Psychological Trauma: Theory, Research, Practice, and Policy, 9, 493-499.

6 Brown, S. M., \& Shillington, A. M. (2017). Childhood adversity and the risk of substance use and delinquency: The role of protective adult relationships. Child Abuse and Neglect, 63, 211-221.

7 Center for Behavioral Health Statistics and Quality. (2016). Key substance use and mental health indicators in the United States: Results from the 2015 National Survey on Drug Use and Health (HHS Publication No. SMA 16-4984, NSDUH Series H-51).

8 Cross, D., Crow, T., Powers, A., \& Bradley, B. (2015). Childhood trauma, PTSD, and problematic alcohol and substance use in low-income, African-American men and women. Child Abuse and Neglect, 44, 26-35.

9 Darke, S., \& Torok, M. (2013). The association of childhood physical abuse with the onset and extent of drug use among regular injecting drug users. Addiction, 109, 610-616.

10 Ding, Y., Lin, H., Zhou, L., Yan, H., \& He, N. (2014). Adverse childhood experiences and interaction with methamphetamine use frequency in the risk of methamphetamine-associated psychosis. Drug and Alcohol Dependence, 142, 295-300.

11 Douglas, K. R., Chan, G., Gelernter, J., Arias, A. J., Anton, R. F., Weiss, R. D., ... Kranzler, H. R. (2010). Adverse childhood events as risk factors for substance dependence: Partial mediation by mood and anxiety disorders. Addictive Behaviors, 35, 7-13.

12 Dube, S. R., Felitti, V. J., Dong, M., Chapman, D. P., Giles, W. H., \& Anda, R. F. (2003). Childhood abuse, neglect, and household dysfunction and the risk of illicit drug use: the adverse childhood experiences study. Pediatrics 111, 564-572.

13 Dubowitz, H., Thompson, R., Arria, A. M., English, D., Metzger, R., \& Kotch, J. B. (2015). Characteristics of Child Maltreatment and Adolescent Marijuana Use. Child Maltreatment, 21, 16-25.

14 Duprey, E. B., Oshri, A., \& Caughy, M. O. (2017). Childhood neglect, internalizing symptoms and adolescent substance use: Does the neighborhood context matter? Journal of Youth and Adolescence, 46, 1582-1597.

15 Elton, A., Smitherman, S., Young, J., \& Kilts, C. D. (2014). Effects of childhood maltreatment on the neural correlates of stress- and drug cue-induced cocaine craving. Addiction Biology, 20, 820-831.

16 Flanagan, J. C., Baker, N. L., Mcrae-Clark, A. L., Brady, K. T., \& Maria, M. M. (2015). Effects of adverse childhood experiences on the association between intranasal oxytocin and social stress reactivity among individuals with cocaine dependence. Psychiatry Research, 229, 94-100.

17 Forster, M., Grigsby, T. J., Rogers, C. J., \& Benjamin, S. M. (2018). The relationship between family-based adverse childhood experiences and substance use behaviors among a diverse sample of college students. Addictive Behaviors, 76, 298-304.

18 Fuller-Thomson, E., Roane, J. L., \& Brennenstuhl, S. (2016). Three types of adverse childhood experiences, and alcohol and drug dependence among adults: An investigation using population-based data. Substance Use and Misuse, 51, 14511461.

19 Goldstein, R. Z., and Volkow, N. D. (2002). Drug addiction and its underlying neurobiological basis: neuroimaging evidence for the involvement of the frontal cortex. Am. J. Psychiatry 159, $1642-1652$.

20 Goldstein, R. Z., and Volkow, N. D. (2011). Dysfunction of the prefrontal cortex in addiction: neuroimaging findings and clinical implications. Nat. Rev. Neurosci. 12, 652-669.

21 Haller, M., \& Chassin, L. (2014). Risk pathways among traumatic stress, posttraumatic stress disorder symptoms: And alcohol and drug problems: A test of four hypotheses. Psychology of Addictive Behaviors, 28, 841-851.

22 Kilpatrick, D. G., Resnick, H. S., Milanak, M. E., Miller, M. W., Keyes, K. M., \& Friedman, M. J. (2013). National estimates of exposure to traumatic events and PTSD prevalence using DSM IV and DSM-5 criteria. Journal of Traumatic Stress, 26, 537-547.

23 Kim, Y. H. (2017). Associations of adverse childhood experiences with depression and alcohol abuse among Korean college students. Child Abuse and Neglect, 67, 338-348.

24 Lawson, K. M., Back, S. E., Hartwell, K. J., Maria, M. M., \& Brady, K. T. (2013). A comparison of trauma profiles among individuals with prescription opioid, nicotine, or cocaine dependence. The American Journal on Addictions, 22, 127131.

25 Le, T. L., Mann, R. E., Levitan, R. D., George, T. P., \& Maunder, R. G. (2016). Sex differences in the relationships 
between childhood adversity, attachment anxiety, and current smoking. Addiction Research and Theory, 25, 146-153.

26 Love, H. A., \& Torgerson, C. N. (2018). Traumatic experiences in childhood and adult substance use in a nonclinical sample: The mediating role of arousal/reactivity. Journal of Marital and Family Therapy, 1, 1-13.

27 Mandavia, A., Robinson, G. G., Bradley, B., Ressler, K. J., \& Powers, A. (2016). Exposure to childhood abuse and later substance use: Indirect effects of emotion dysregulation and exposure to trauma. Journal of Traumatic Stress, 29, 422-429.

28 Mokdad, A. H., Marks, J. S., Stroup, D. F., \& Gerberding, J. L. (2004). Actual causes of death in the United States, 2000. Journal of the American Medical Association,291, 1238-1245.

29 Myers, B., Mclaughlin, K. A., Wang, S., Blanco, C., \& Stein, D. J. (2014). Associations between childhood adversity, adult stressful life events, and past-year drug use disorders in the National Epidemiological Study of Alcohol and Related Conditions (NESARC). Psychology of Addictive Behaviors, 2, 1117-1126.

30 Oshri, A., Carlson, M. W., Kwon, J. A., Zeichner, A., \& Wickrama, K. K. (2016). Developmental growth trajectories of self-esteem in adolescence: Associations with child Neglect and drug use and abuse in young adulthood. Journal of Youth and Adolescence, 46, 151-164.

31 Proctor, L. J., Lewis, T., Roesch, S., Thompson, R., Litrownik, A. J., English, D., Dubowitz, H. (2017). Child maltreatment and age of alcohol and marijuana initiation in high-risk youth. Addictive Behaviors, 75, 64-69.

32 Quinn, K., Boone, L., Scheidell, J. D., Mateu-Gelabert, P., Mcgorray, S. P., Beharie, N. \& Khan, M. R. (2016). The relationships of childhood trauma and adulthood prescription pain reliever misuse and injection drug use. Drug and Alcohol Dependence, 169, 190-198.

33 Sartor, C. E., Bachrach, R. L., Stepp, S. D., Werner, K. B., Hipwell, A. E., \& Chung, T. (2017). The relationship between childhood trauma and alcohol use initiation in Black and White adolescent girls: Considering socioeconomic status and neighborhood factors. Social Psychiatry and Psychiatric Epidemiology, 53, 21-30.

34 Shin, S. H., Hong, H. G., \& Hazen, A. L. (2010). Childhood sexual abuse and adolescent substance use: A latent class analysis. Drug and Alcohol Dependence, 109, 226-235.

35 Shin, S. H., Miller, D. P., \& Teicher, M. H. (2013). Exposure to childhood neglect and physical abuse and developmental trajectories of heavy episodic drinking from early adolescence into young adulthood. Drug and Alcohol Dependence, 127, 3138.

36 Stein, M. D., Conti, M. T., Kenney, S., Anderson, B. J., Flori, J. N., Risi, M. M., \& Bailey, G. L. (2017). Adverse childhood experience effects on opioid use initiation, injection drug use, and overdose among persons with opioid use disorder. Drug and Alcohol Dependence, 179, 325-329.

37 Substance Abuse and Mental Health Services Administration. (2013). Results from the 2012 national survey on drug use and health: Summary of national findings (NSDUH Series H-46, HHS Publication No. SMA 13-4795). Rockville, MD: Author.

38 Taplin, C., Saddichha, S., Li, K., \& Krausz, M. R. (2014). Family history of alcohol and drug abuse, childhood trauma, and age of first drug injection. Substance Use and Misuse, 49, 1311-1316.

39 Tonmyr, L., \& Shields, M. (2017). Childhood sexual abuse and substance abuse: A gender paradox? Child Abuse and Neglect, 63, 284-294.

40 U.S. Department of Health and Human Services, Administration for Children and Families, Administration on Children, Youth and Families, Children's Bureau (2015). Child maltreatment 2013

41 U.S. Department of Health and Human Services, \& Administration for Children and Families, Administration on Children, Youth and Families, Children's Bureau. (2017). Child Maltreatment 2015

42 Verona, E., Murphy, B., \& Javdani, S. (2016). Gendered pathways: Violent childhood maltreatment, sex exchange, and drug use. Psychology of Violence, 6, 124-134.

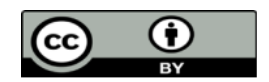

This work is licensed under Creative Commons Attribution 4.0 License
To Submit Your Article Click Here: Submit Manuscript

DOI: $10.31579 / 2690-8794 / 019$
Ready to submit your research? Choose Auctores and benefit from:

* fast, convenient online submission

* rigorous peer review by experienced research in your field

* rapid publication on acceptance

* authors retain copyrights

* unique DOI for all articles

* immediate, unrestricted online access

At Auctores, research is always in progress.

Learn more www.auctoresonline.org/journals/clinical-medical-reviewsand-reports 